\title{
Gene expression analysis in the parvalbumin- immunoreactive PV1 nucleus of the mouse lateral hypothalamus
}

\author{
F. Girard,${ }^{1}$ Z. Meszar, ${ }^{1}$ C. Marti, ${ }^{1}$ F. P. Davis ${ }^{2}$ and M. Celio ${ }^{1}$ \\ ${ }^{1}$ Division of Anatomy and Program in Neuroscience, Université de Fribourg, Route A. Gockel 1, CH1700 Fribourg, Switzerland \\ 2Janelia Farm Research Campus, Howard Hughes Medical Institute, Ashburn, VA, USA
}

Keywords: brain, calcium-binding protein, glutamate, lateral tuberal nucleus

\begin{abstract}
A solitary, elongated cluster of parvalbumin-immunoreactive neurons has been previously observed in the rodent ventrolateral hypothalamus. However, the function of this so-called PV1 nucleus is unknown. In this article, we report the results of an unbiased, broad and in-depth molecular characterization of this small, compact group of neurons. The Allen Brain Atlas database of in situ hybridization was screened in order to identify genes expressed in the PV1-nucleus-containing area of the hypothalamus, and those that might be co-expressed with parvalbumin. Although GABA is the principal neurotransmitter in parvalbumin-expressing cells in various other brain areas, we found that PV1 neurons express the vesicular glutamate transporter (VGlut) VGlut2-encoding gene Slc17a6 and are negative for the glutamic acid decarboxylase 1 (GAD1) gene. These cells also express the mRNA for the neuropeptides Adcyap1 and possibly Nxph4, express several types of potassium and sodium channels, are under the control of the neurotransmitter acetylcholine, bear receptors for the glial-derived neurotrophic factor, and produce an extracellular matrix rich in osteopontin. The PV1 nucleus is thus composed of glutamatergic nerve cells, expressing some typical markers of long-axon, projecting neurons (e.g. VGlut2), but also co-expressing genes typical of short-axon GABA neurons (e.g. a variety of potassium channels). Hence, neurons of the PV1 nucleus combine physiological characteristics of interneurons with those of projection neurons.
\end{abstract}

\section{Introduction}

The lateral hypothalamic area (LHA) is a large and heterogeneous region, containing several distinct nuclear groups. Although the LHA was historically described as a 'feeding center', its connectivity with autonomic, sympathetic and mesolimbic systems indicates that it regulates the physiological responses to a range of stimuli (such as food, drugs or stress) (reviewed in Berthoud \& Münzberg, 2011). Interest in the LHA has recently re-emerged with the discovery of the orexin/hypocretin system and its importance in regulating the sleep/wake cycle (reviewed in Sakurai et al., 2010; Leinninger, 2011). The complex structural organization of the LHA (dense and complex array of cell groups and fiber pathways) hampers an analysis of the anatomical to functional relationships. Such an analysis would be aided by a better characterization of the neurotransmitter and neuropeptide content of its neurons.

The EF-hand calcium-binding protein parvalbumin (Pvalb) is strongly expressed in various regions of the postnatal rodent brain, including the cortex, hippocampus, thalamus, midbrain and cerebellum (Celio, 1990; Schwaller, 2009). On the basis of $\mathrm{Ca}^{2+}$-binding and release kinetics, Pvalb acts as a slow calcium buffer, playing important roles in modulating intracellular calcium dynamics in neurons

(Baimbridge et al., 1992; Schwaller, 2009). A solitary cluster of Pvalb-immunoreactive neurons, referred to as the PV1 nucleus, has been observed in the ventrolateral division of the medial forebrain bundle of the rodent hypothalamus (Celio, 1990). These neurons are orientated horizontally, and have a bipolar or multipolar form (Meszar et al., 2011). Unlike Pvalb-immunoreactive neurons in most other regions of the brain, those of the PV1 nucleus lack the GABAsynthesizing enzyme GAD and GABA itself, but are immunoreactive for glutamate (Meszar et al., 2011).

Topographical mapping of the gene expression patterns in the mammalian central nervous system is crucial for an understanding of the functional networks. During the past few years, research in the field of neuroscience has entered a new era with the release of in situ hybridization (ISH) data at the genomic scale, such as appear in the Allen Brain Atlas (ABA) for mouse gene expression (Lein et al., 2007). Indeed, such data provide powerful tools allowing a systematic analysis of particular neuroanatomical structures at the gene expression level (D'Souza et al., 2008; Olszewski et al., 2008; Thompson et al., 2008; Dong et al., 2009).

In this context, identifying genes co-expressed with Pvalb may yield further insight into the evolution and function of the PV1 nucleus. The microarray approach has proved to be an efficient method for genomewide expression profiling, but it is a challenging technique for structures that embrace only a small number of cells, such as the PV1 nucleus. Therefore, we undertook a genome-scale ISH analysis to 
identify genes co-expressed with Pvalb in PV1 neurons. For this purpose, the ABA database was screened to pinpoint potential candidates, and ISH was used to demonstrate co-expression with Pvalb.

\section{Materials and methods \\ Animals}

C57BL/6J mice were used in this study. Animals (20-30 g in weight) were anesthetized with pentobarbital $(100 \mathrm{mg} / \mathrm{kg})$, and perfused with ice-cold $0.9 \% \mathrm{NaCl}$. Brains were then removed, immediately frozen on dry ice and stored at $-70{ }^{\circ} \mathrm{C}$ until use. The project was approved by the veterinary committee for animal research of the Canton of Fribourg, Switzerland.

\section{In situ hybridization and immunostaining}

Digoxygenin-labeled RNA probes were prepared following recommendations from Roche (DIG application manual for non radioactive in situ hybridization, 3rd edn). Total RNA from mouse brain (Zyagen, San Diego, CA, USA) was reverse transcribed using oligodT as primer, and this reaction mixture was used as the template for polymerase chain reactions using primers specific for each of the genes tested, as described in the ABA (http://www.brain-map.org/). Forward and reverse primers were flanked by T3 and SP6 RNA polymerase core promoter sequences, respectively (T3, AATTAACCCTCACTAAAGGG and SP6, GCGATTTAGGTGACACTATAG). All primers were purchased from Microsynth (Balgach, Switzerland). Antisense and sense RNA probes were prepared from each specific polymerase chain reaction template by in vitro transcription with either SP6 or T3 RNA polymerase and digoxygenin-labeled UTP (Roche Applied Science, Switzerland). Sense probes were used as the negative control of the hybridization procedure. The quality and yields of the probes were estimated on agarose gel. For Pvalb RNA probe, polymerase chain reaction was performed directly using mouse Pvalb full-length cDNA (purchased from ImaGenes, Berlin, Germany) as template, using the Pvalbspecific primers described in the ABA.

For Macaca fascicularis probes, the following primers were used in reverse transcription-polymerase chain reaction experiments using total RNA from Macaca brain (a gift from Dr F. Raulf, Novartis, Basel, Switzerland): Serpini1 (forward, ATGTGATGAAAATTGCCAAT; reverse, ATTCCTGAGACAGCAGCAGC) and Slc17a7 (forward, ATGGGCCCCACCCTTAGAAC; reverse, CTAAACTTCGTGAGGGGGTT). Primers were flanked by a T3 (forward) and SP6 (reverse) RNA polymerase core promoter sequence as described for mouse probes. Probes were made exactly as described for mouse.

The $12-14 \mu \mathrm{m}$ coronal brain cryosections were prepared on Superfrost gold slides (Medite, Nunningen, Switzerland), and stored at $-70{ }^{\circ} \mathrm{C}$ until use. ISH was performed essentially following the recommendations of Roche. Briefly, sections were fixed in $4 \%$ paraformaldehyde in phosphate-buffered saline for $20 \mathrm{~min}$ at $4{ }^{\circ} \mathrm{C}$, rinsed in phosphate-buffered saline, and incubated for $10 \mathrm{~min}$ in $0.1 \mathrm{M}$ triethanolamine containing $0.25 \%$ acetic anhydride. Sections were sequentially incubated in phosphate-buffered saline $/ 0.1 \mathrm{M}$ glycine $/ 0.3 \%$ Triton-X100 for $5 \mathrm{~min}$, phosphate-buffered saline for $5 \mathrm{~min}, 0.1 \mathrm{M}$ Tris, $\mathrm{pH} 7.5 / 50 \mathrm{~mm}$ EDTA containing $1 \mu \mathrm{g} / \mathrm{mL}$ proteinase $\mathrm{K}$ at $37{ }^{\circ} \mathrm{C}$ for $3-5 \mathrm{~min}$, and postfixed for $5 \mathrm{~min}$ in $4 \%$ paraformaldehyde (PFA). Sections were prehybridized for $30 \mathrm{~min}$ at $57{ }^{\circ} \mathrm{C}$ in $50 \%$ formamide $/ 5 \times$ saline sodium citrate buffer (SSC), then hybridized for $16 \mathrm{~h}$ at $57{ }^{\circ} \mathrm{C}$ with the respective probes in buffer containing $50 \%$ formamide/5× SSC/10\% Dextran sulfate/1× Denhardt's solution $/ 2.5 \mathrm{mg} / \mathrm{mL}$ Torula RNA. Sections were washed at the hybridization temperature in, respectively, $2 \times \mathrm{SSC}, 1 \times \mathrm{SSC}$ and $0.2 \times \mathrm{SSC}, 20 \mathrm{~min}$ each, then incubated for $30 \mathrm{~min}$ in buffer $1(0.1 \mathrm{M}$ Tris, $\mathrm{pH} 7.5 / 0.15 \mathrm{M} \mathrm{NaCl}$ ) containing $0.1 \%$ Triton-X100 and $1 \%$ Roche blocking reagent. Alkaline-phosphatase-conjugated anti-digoxygenin (Roche Applied Science) was applied for $2 \mathrm{~h}$. Sections were washed three times for $15 \mathrm{~min}$ in buffer 1 , and $15 \mathrm{~min}$ in buffer 2 (0.1 M Tris, $\mathrm{pH}$ 9.5/0.1 M NaCl/50 mM $\mathrm{MgCl}_{2}$ ). Detection was performed with nitroblue tetrazolium (NBT)-5-bromo-4-chloro-3'indolylphosphate (BCIP) (Roche Applied Science) in buffer 2, in the presence of $100 \mathrm{~mm}$ levamisole.

Immunostaining with rabbit anti-Pvalb (Swant, Marly, Switzerland) on $20 \mu \mathrm{m}$ coronal sections was performed following the standard protocol.

\section{Informatics}

ALLENMINER (v1.5; Davis \& Eddy, 2009) was used to retrieve threedimensional expression files from the ABA (Lein et al., 2007) at $100 \mu \mathrm{m}$ resolution (XPR files; $n=21741$ ) and smoothed $200 \mu \mathrm{m}$ resolution (SVA files; $n=24$ 858) formats (January 2010). A region of interest encompassing the PV1 nucleus was first defined in the ABA coordinate system by specifying those voxels in a cuboid [coronal $x=(56,71)$, horizontal $y=(60,67)$, sagittal $z=(42,48)]$ that did not overlap with the tuberal nucleus, subthalamic nucleus, or anterior hypothalamic nucleus, as defined by the ABA reference atlas. One layer of edge voxels that bordered the tuberal nucleus, subthalamic nucleus, or anterior hypothalamic nucleus structures was removed to reduce the effect of mis-registration artifacts, which especially affect small regions (Davis \& Eddy, 2009), yielding a final PV1 region of interest containing 699 voxels (of $100 \mu \mathrm{m}$ resolution).

ALLENMINER queries were then performed to identify (i) genes that are locally enriched in the PV1 region of interest, and (ii) genes whose PV1 expression is similar to Pvalb. First, the ABA data $\left(100 \mu \mathrm{m}^{3}\right.$ format) were evaluated for enrichment of gene expression in the PV1 region of interest relative to its local neighborhood, defined by a 4voxel-thick shell whose PV1-bordering layer is removed (run modes roiseparate_adjacent, roi_list, calcentropy_xpr_roi_results). Second, the similarity of each expression dataset (200 $\mu \mathrm{m}$ format) to each Pvalb expression series (coronal, $n=2$; sagittal, $n=19$ ) was quantified by the Pearson's correlation coefficient of expression levels across all voxels in the region of interest where expression was registered for both genes (run mode expr_sim_query).

The accuracies of the searches were assessed using a benchmark set of genes $(n=21)$ whose Pvalb co-expression was independently determined by ISH. When thresholded to avoid all non-PV1expressing benchmark genes $(n=4)$, the enrichment and similarity queries achieve 50 and $60 \%$ true positive rates and predict 584 and 326 PV1-expressing genes, respectively. Combining the results predicts 843 genes, at an estimated $81 \%$ true positive rate. Requiring detection by both search strategies predicts 67 genes, at an estimated $25 \%$ true positive rate.

\section{Results \\ Computational analysis of the parvalbumin-immunoreactive PV1 nucleus of the lateral hypothalamus}

The ABA ISH database covers over 21000 genes expressed in the adult mouse brain (Lein et al., 2007), and is an invaluable tool for mapping gene expression patterns in specific brain areas (D’Souza 
TABLE 1. List of the genes selected after screening the ABA for expression in the PV1 region

\begin{tabular}{|c|c|c|}
\hline Gene & Complete name & Gene ontology \\
\hline Adcy 2 & Adenylate cyclase 2 & Adenylate cyclase activity \\
\hline Adcyap 1 & Adenylate cyclase-activating polypeptide 1 & Neuropeptide hormone activity \\
\hline Anxa5 & Annexin A5 & Calcium-dependent phospholipid binding \\
\hline Asb13 & Ankyrin repeat and SOCS box-containing protein 13 & Unknown \\
\hline Cart & Cocaine- and amphetamine-regulated transcript & Neuropeptide/hormone activity \\
\hline Cdh13 & Cadherin 13 & Cell adhesion \\
\hline $\mathrm{Cdh} 23$ & Cadherin 23 (otocadherin) & Cell adhesion \\
\hline Chrm2 & Cholinergic receptor, muscarinic 2 , cardiac & Muscarinic acetylcholine receptor activity \\
\hline Chrm3 & Cholinergic receptor, muscarinic 3 , cardiac & Muscarinic acetylcholine receptor activity \\
\hline Chrnb2 & Cholinergic receptor, nicotinic, beta polypeptide 2 (neuronal) & Nicotinic acetylcholine receptor activity \\
\hline Col11a1 & Procollagen, type XI, alpha 1 & ECM constituent \\
\hline Crh & Corticotropin-releasing hormone & Hormone activity \\
\hline Crhr1 & Corticotropin-releasing hormone receptor 1 & Corticotropin-releasing factor receptor activity \\
\hline Crtac1 & Cartilage acidic protein 1 & ECM constituent \\
\hline Dab1 & Disabled homolog 1 & Signal transduction \\
\hline Fndc5 & Fibronectin type III domain-containing 5 & Unknown \\
\hline Gad1 & Glutamic acid decarboxylase 1 & Glutamate decarboxylase activity \\
\hline Gfra1 & Glial cell line-derived neurotrophic factor family receptor alpha 1 & Receptor activity \\
\hline Grm1 & Glutamate receptor, metabotropic 1 & Glutamate receptor activity \\
\hline Hen 2 & Hyperpolarization-activated, cyclic nucleotide-gated $\mathrm{K}{ }^{+} 2$ & Potassium channel activity \\
\hline Kcna1 & Potassium voltage-gated channel, shaker-related subfamily, member 1 & Potassium channel activity \\
\hline Kcna2 & Potassium voltage-gated channel, shaker-related subfamily, member 2 & Potassium channel activity \\
\hline Kcnab2 & Potassium voltage-gated channel, shaker-related subfamily, beta member 2 & Potassium channel activity \\
\hline Kcnab3 & Potassium voltage-gated channel, shaker-related subfamily, beta member 3 & Potassium channel activity \\
\hline Kenc1 & Potassium voltage-gated channel, Shaw-related subfamily, member 1 & Potassium channel activity \\
\hline Kenc2 & Potassium voltage-gated channel, Shaw-related subfamily & Potassium channel activity \\
\hline Kcnk1 & Potassium channel, subfamily $\mathrm{K}$, member 1 & Potassium channel activity \\
\hline Lgi2 & Leucine-rich repeat LGI family, member 2 & Unknown \\
\hline Lhx1 & LIM homeobox protein 1 & Transcription factor \\
\hline Limk1 & LIM-domain-containing, protein kinase & Protein kinase activity \\
\hline Lynx1 & Ly6/neurotoxin 1 & Acetylcholine receptor inhibitor activity \\
\hline Lynx2 & Ly6/Plaur domain-containing 1 & Acetylcholine receptor inhibitor activity \\
\hline Mfap5 & Microfibrillar-associated protein 5 & ECM constituent \\
\hline Nefh & Neurofilament, heavy polypeptide & Neurofilament cytoskeleton organization \\
\hline Nefl & Neurofilament, light polypeptide & Neurofilament cytoskeleton organization \\
\hline Nef3 & Neurofilament 3 , medium & Neurofilament cytoskeleton organization \\
\hline Nos1 & Nitric oxide synthase 1 , neuronal & Nitric oxide synthase activity \\
\hline Ntng1 & Netrin G1 & ECM interaction/axonogenesis \\
\hline Nxph4 & Neuroexophilin 4 & Receptor binding \\
\hline Oprm1 & Opioid receptor, mu 1 & Neuropeptide receptor activity \\
\hline Pdyn & Prodynorphin & Neuropeptide/hormone activity \\
\hline Penk1 & Preproenkephalin 1 & Opioid peptide activity \\
\hline Pib5pa & Phosphatidylinositol $(4,5)$ bisphosphate 5-phosphatase, A & $\begin{array}{l}\text { Phosphatidylinositol-3,4,5-trisphosphate } \\
\text { 5-phosphatase activity }\end{array}$ \\
\hline Pitx2 & Paired-like homeodomain transcription factor 2 & Transcription factor \\
\hline Plcb4 & Phospholipase beta 4 & Phospholipase activity \\
\hline Pnkd & Paroxysmal non-kinesiogenic dyskinesia & Hydroxylase activity \\
\hline Ret & Ret proto-oncogene & $\begin{array}{l}\text { Transmembrane receptor protein tyrosine } \\
\text { kinase activity }\end{array}$ \\
\hline Sall3 & Sal-like 3 & Transcription factor \\
\hline Scg2 & Secretogranin II & Cytokine activity \\
\hline Scn1a & Sodium channel, voltage-gated, type I, alpha & Sodium channel activity \\
\hline $\mathrm{Scn} 4 \mathrm{~B}$ & Sodium channel, type IV, beta & Sodium channel activity \\
\hline Scrt1 & Scratch homolog 1, zinc finger protein (Drosophila) & Transcription factor \\
\hline $\mathrm{Sh} 3 \mathrm{gl} 2$ & SH3-domain GRB2-like 2 & Unknown \\
\hline Sema3f & $\begin{array}{l}\text { Sema domain, immunoglobulin domain, short basic domain, secreted, } \\
\text { (semaphorin) 3f }\end{array}$ & Chemorepellent, receptor activity \\
\hline Serpini1 & Serine (or cysteine) peptidase inhibitor, clade I, member 1 & Peptidase inhibitor activity \\
\hline Slc17a6 & Solute carrier family 17, member 6 (VGlut2) & Neurotransmitter (glutamate) transport \\
\hline Slc17a7 & Solute carrier family 17 , member 7 (VGlut1) & Neurotransmitter (glutamate) transport \\
\hline Sncg & Synuclein gamma & Unknown \\
\hline Spp1 & Secreted phosphoprotein 1 & Cytokine activity. ECM binding \\
\hline Stxbp6 & Syntaxin-binding protein (amysin) & Vesicle-mediated transport \\
\hline Tac1 & Tachykinin 1 & Neuropeptide activity \\
\hline Tac1r & Tachykinin receptor 1 & Neuropeptide receptor activity \\
\hline Tac2 & Tachykinin 2 & Neuropeptide activity \\
\hline Tmem163 & Transmembrane protein 163 & Unknown \\
\hline Trh & Thyrotropin-releasing hormone & Hormone activity \\
\hline Uqcrh & Ubiquinol-cytochrome $\mathrm{c}$ reductase hinge protein & Ubiquinol-cytochrome $\mathrm{c}$ reductase activity \\
\hline
\end{tabular}


TABLE 1. (Continued)

\begin{tabular}{lll}
\hline Gene & Complete name & Gene ontology \\
\hline Vamp1 & Vesicle-associated membrane protein 1 & Vesicle-mediated transport \\
LOC382102 & Gene model 1125 & Unknown \\
C920006C10Rik & RIKEN cDNA C920006C10 gene (EFR3-like) & Unknown \\
A930038C07Rik & RIKEN cDNA A930038C07 gene & Heparin binding, ECM organization \\
6330403A02Rik & RIKEN cDNA 6330403A02 gene & Pnknown \\
LOC433022 & Plcxd2, phosphatidylinositol-specific phospholipase C, X domain-containing 2 \\
2300002D11Rik & RIKEN cDNA 2300002D11 gene (Trnp1) & Unknown \\
4930544G21Rik & RIKEN cDNA 4930544G21 gene (Sphkap) & Unknown \\
4930572J05Rik & RIKEN cDNA 493057J05 gene & Unknown \\
9130213B05Rik & RIKEN cDNA 9130213B05 gene (Parm1) & \\
\hline
\end{tabular}

Given are the abbreviated and complete names, and known or proposed function (Gene Ontology) for the candidate genes selected in this study.

et al., 2008; Olszewski et al., 2008; Thompson et al., 2008; Dong et al., 2009). Initially, we screened the ABA database for genes that are regionally enriched in the lateral hypothalamus, using the provided tools: (i) the anatomic search tool focusing on the hypothalamus; (ii) the gene finder tool of the Anatomic Gene Expression Atlas focusing on the lateral hypothalamus; and (iii) the neuroblast tool searching for genes with an expression pattern similar to that of Pvalb. This first round of screening was then followed by an automated ALLENMINER search (Davis \& Eddy, 2009) for genes that are either enriched in the PV1 nucleus, or that exhibit a Pvalb-like expression pattern (see Materials and methods). From these various screens, we chose to analyze in more detail a list of 76 candidate genes expressed in the region containing the PV1 nucleus (see below). These can be ontologically classified as follows (see Table 1): ion channel activity: sodium (Scn1a, Scn4b), potassium (Hen2, Kena1, Kcna2, Kcnab2, Kcnab3, Kcnc1, Kcnc2, Kcnk1); transporter activity: Slc17a6, Slc17a7, Stxbp6, Vamp1; enzyme activity: Adcy2, Gad1, Limk1, Nos1, Pib5pa, Plcb4, Plcxd2, Pnkd, Uqcrh; receptor activity: Chrm2, Chrm3, Chrnb2, Crhr1, Gfra1, Grm1, Oprm1, Ret, Sema3f, Tac1r; transcription factor activity: Lhx1, Pitx2, Sall3, Scrt1; neuropeptide/neurohormone activity: Adcyap1, Cart, Nxph4, Crh, Pdyn, Penk1, Tac1, Tac2, Trh; cytoskeleton structural components: Nefh, Nef3, Nefl; extracellular matrix (ECM)/cell adhesion molecules: Cdh13, Cdh23, Col11a1, Crtac1, Mfap5, Ntgn1, Spp1, A930038C07Rik; receptor inhibitor activity: Lynx1, Lynx2; peptidase inhibitor activity Serpini1; miscellaneous: Anxa5, Dab1, Scg2; and unknown function: Asb13, EFR3like, Fndc5, Lgi2, Parm1, Sh3g12, Sncg, Sphkap, Trnp1, Tmem163, LOC382102, 4930572J05Rik,
63304003A02Rik. The complete list of 843 candidate genes identified with the ALLENMINER search is available as Supporting Information (Tables S1-S4).

\section{Co-expression with parvalbumin in the PV1 nucleus}

In the mouse brain, the PV1 nucleus can be revealed by either ISH to Pvalb mRNA, or Pvalb immunostaining (Fig. 1) (Meszar et al., 2011). In the $\mathrm{ABA}$, it lies between coronal levels 68 and 72, forming an elongated, longitudinally orientated structure within the lateral hypothalamus (Fig. 2G) (Meszar et al., 2011). Typically, between 5 and 10 Pvalb-positive cells are visible on each coronal section (Fig. 1). We thus reasoned that ISH on adjacent $12-14-\mu \mathrm{m}$-thick coronal cryosections would resolve a sufficient number of cells co-expressing Pvalb and the candidate genes. Using the selection criteria: (i) a restricted expression pattern; (ii) a good signal-to-noise ratio of the antisense RNA probe; and (iii) a potential functional importance, 27 of the 76 candidate genes were tested by ISH. Among them, 19 were shown here to be co-expressed with Pvalb in the same neurons, whereas 8 were expressed by intermingling Pvalb-negative cells. The results are presented in Figs 2 and 3, and summarized in Table 2. Figure 2 depicts the co-expression in some PV1 neurons of Pvalb with three candidate genes, Vamp1, Adcyap1 and Slc17a6 (solute carrier family 17 , member 6 , encoding the glutamate transporter VGlut2). Figure 3 further presents co-expression with Pvalb of several additional genes: Serpini1, Spp1, Pib5pa, Nefl, Asb13, Hen2, Kcna2, Kcnc1 and Scn4b. The co-expression was not apparent within all of the Pvalb-positive cells. This incomplete co-existence was perhaps not surprising, as we

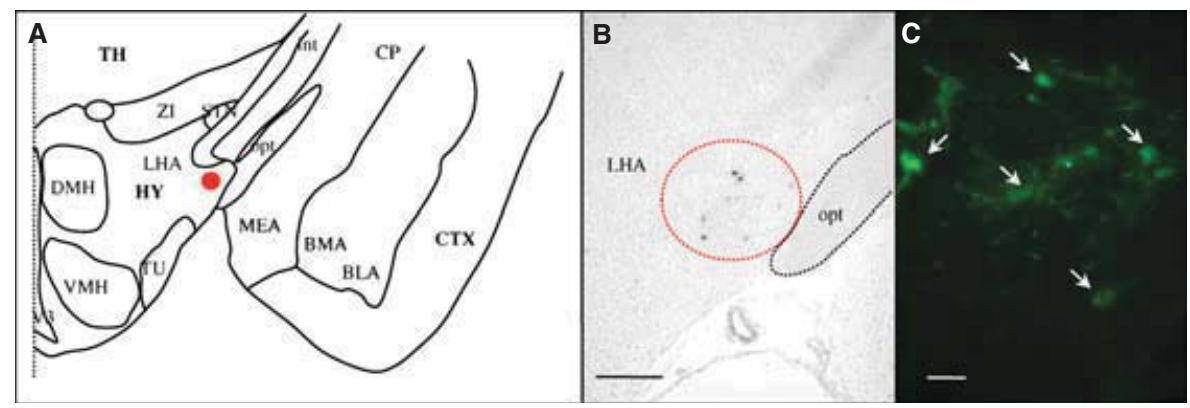

FIG. 1. Localization of the PV1 nucleus within the LHA in the adult mouse brain. (A) Schematic representation of brain areas at coronal level 71, according to the Allen Reference Atlas. BLA, basolateral amygdalar nucleus; BMA, basomedial amygdalar nucleus; CP, caudoputamen; CTX, cerebral cortex; DMH, dorsomedial hypothalamic nucleus; HY, hypothalamus; int, internal capsule; MEA, medial amygdalar nucleus; opt, optic tract; STN, subthalamic nucleus; TH, thalamus; TU, tuberal nucleus; V3, third ventricle; VMH, ventromedial hypothalamic nucleus; ZI, zona incerta. The red point indicates the location of the PV1 nucleus at this coronal level. (B) Pvalb mRNA expression, and (C) Pvalb immunoreactivity in the PV1 nucleus. The PV1 nucleus is highlighted with a red circle in A and B. White arrows point to cell bodies immunoreactive for Pvalb. Scale bar: $200 \mu \mathrm{m}$ (B), $20 \mu \mathrm{m}(\mathrm{C})$. For interpretation of color references in figure legend, please refer to the Web version of this article. 

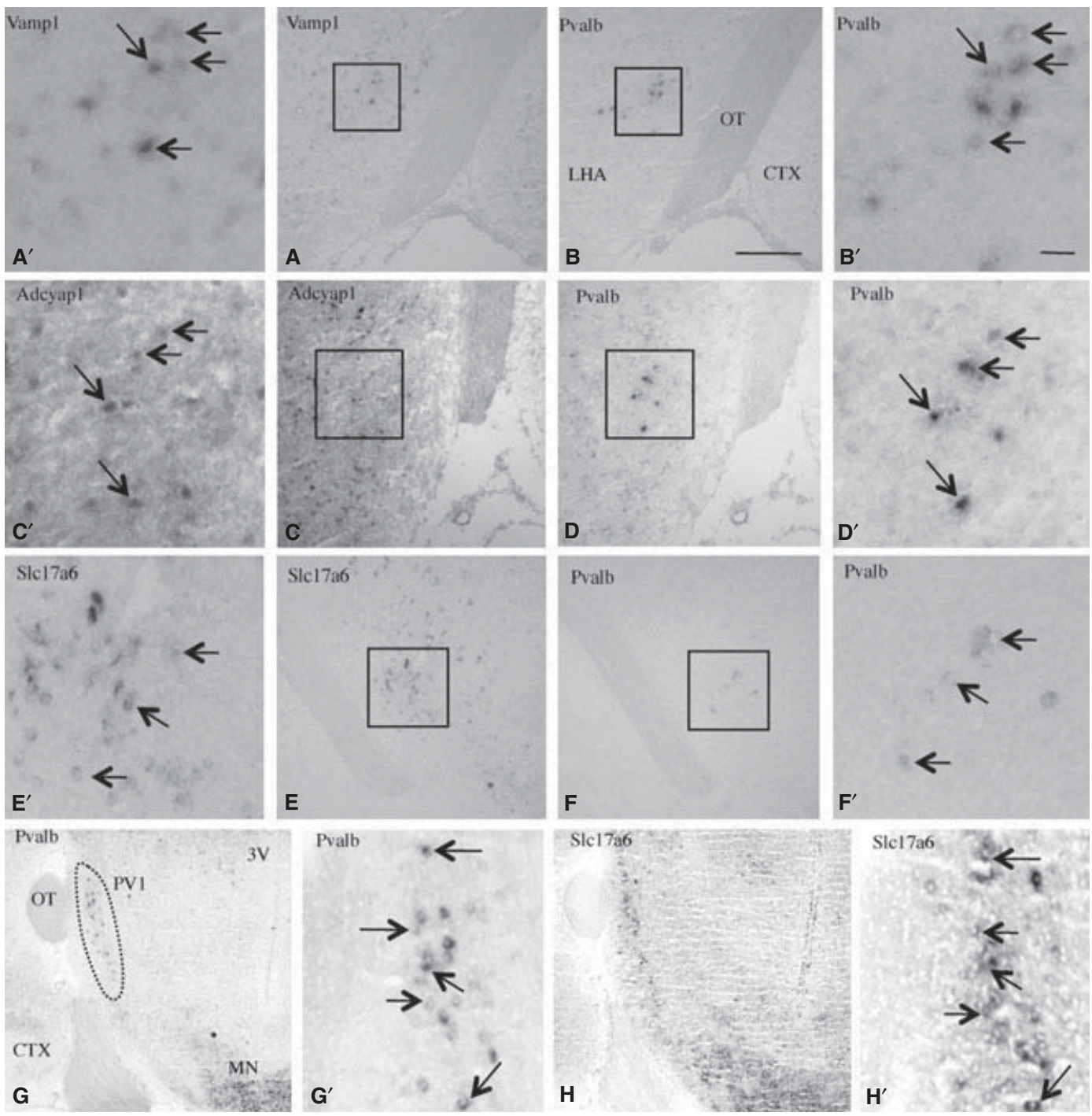

FIG. 2. Expression of several genes in Pvalb-expressing cells of the PV1 nucleus. A/B, C/D and E/F are adjacent coronal brain sections stained by ISH for Vamp1 (A and $\mathrm{A}^{\prime}$ higher magnification), Adcyap1 (C and $\left.\mathrm{C}^{\prime}\right)$, Slc17a6 (E and $\mathrm{E}^{\prime}$ ) and Pvalb (B/B', D/D' and F/F'). Square delineates the PV1 nucleus shown at higher magnification in $\mathrm{A}^{\prime}-\mathrm{F}^{\prime}$. Adjacent horizontal brain sections stained for Pvalb ( $\mathrm{G}$ and higher magnification $\left.\mathrm{G}^{\prime}\right)$ and Slc17a6 (H and $\left.\mathrm{H}^{\prime}\right)$. Scale bar: $200 \mu \mathrm{m}(\mathrm{B}), 20 \mu \mathrm{m}$ $\left(\mathrm{B}^{\prime}\right)$. Black arrows point to cells co-expressing Pvalb and the tested genes. CTX, cerebral cortex; MN, mammillary nucleus; OT, optic tract; PV1, PV1 nucleus, highlighted by a dashed line in $\mathrm{G} ; 3 \mathrm{~V}$, third ventricle.

used 12-14- $\mu \mathrm{m}$-thick adjacent sections. Indeed, given the size of the Pvalb-immunoreactive cell bodies (20-30 $\mu \mathrm{m})$ (Meszar et al., 2011), not all of the cell bodies were present on both of the adjacent sections, explaining the lack of complete co-expression, and making a quantification analysis impossible with this technique. However, the possibility that some genes are preferentially expressed in certain neuronal subpopulations but not in others cannot be excluded. This point will need to be clarified by immunohistochemistry.

Previous immunohistochemical studies suggested that PV1 neurons were negative for GABA, and positive for glutamate (Meszar et al., 2011). The present study confirms these observations, as these cells express Slc17a6/VGlut2, but not Gad1. The expression of VGlut2 is sufficient to define a glutamatergic phenotype in neurons (Takamori et al., 2000). Hence, our data indicate that Pvalb-expressing cells of the PV1 nucleus are glutamatergic, excitatory neurons. Not surprisingly, Slc17a7, which encodes the other vesicular glutamate transporter, VGlut1, is not expressed in PV1 cells (Table 2). Most glutamatergic neurons are indeed known to express one of the two transporters, and these cell populations are probably functionally different.
The LHA harbors several neuronal subpopulations expressing various neuropeptides, such as the orexigenic peptides galanin, orexin/hypocretin, melanin-concentrating hormone, and the anorexigenic peptides neurotensin and Cart (for review, see Leinninger, 2011). Several genes encoding hypothalamic neuropeptides were pinpointed during our screening process. As shown in Fig. 4, although the neurons expressing these peptides sometimes abutted on, or even intermingled with, the PValb-expressing neurons, cells that co-expressed Pvalb and one of the tested genes (Tac1 and Tac2, Penk1, Pdyn, Trh, and Cart) were never revealed. Similarly, Pvalb-positive neurons of the PV1 nucleus were never observed to co-express orexin/hypocretin, galanin or melanin-concentrating hormone (Meszar et al., 2011).

\section{Co-expression with parvalbumin in other brain areas}

Next we looked at whether these genes expressed in the PV1 nucleus were also present in Pvalb neurons in other regions of the brain. Closer examination at Pvalb-expressing cells in GABAergic neurons of the reticular nucleus of the thalamus and the Pvalb-positive basket cells in 
TABLE 2. Summary of co-expression with Pvalb in the hypothalamic PV1 nucleus, thalamic reticular nucleus (RT) and hippocampal dentate gyrus (DG)

\begin{tabular}{|c|c|c|c|c|c|}
\hline \multirow[b]{2}{*}{ Gene } & \multicolumn{3}{|c|}{ Co-expression with Pvalb in } & \multirow{2}{*}{\multicolumn{2}{|c|}{$\begin{array}{l}\text { Possible co-expression with Pvalb in } \\
\text { Stratum oriens and molecular layer of } \\
\text { the cerebellum }\end{array}$}} \\
\hline & PV1 nucleus & DG & RT & & \\
\hline Adcyap1 & Yes & $\mathrm{NE}$ & $\mathrm{NE}$ & $N E$ & $N E$ \\
\hline Asb13 & Yes & nt (probable) & nt (probable) & Yes (few) & $N E$ \\
\hline Cart & No & $\mathrm{NE}$ & $\mathrm{NE}$ & $N E$ & $N E$ \\
\hline Crhr1 & Yes & $\mathrm{NE}$ & Yes & $N E$ & $N E$ \\
\hline Gad1 & No & Yes & Yes & Yes & Yes \\
\hline Gfra1 & Yes & Yes & Yes & Yes & $N E$ \\
\hline $\operatorname{Hen} 2$ & Yes & nt (probable) & Yes & Yes (few) & $N E$ \\
\hline Kena2 & Yes & Yes & Yes & Yes & Yes \\
\hline Kenc1 & Yes & Yes & Yes & Yes & $N E$ \\
\hline Lynx1 & Yes & No & Yes & Yes & Yes \\
\hline Lynx2 & Yes & No & No & $N E$ & $N E$ \\
\hline Nefh & Yes & nt (probable) & Yes & Yes & Yes (few) \\
\hline Nefl & Yes & Yes & Yes & Yes (few) & Yes (few) \\
\hline Nef3 & Yes & nt (probable) & Yes & Yes (few) & Yes (few) \\
\hline Pdyn & No & $\mathrm{NE}$ & $\mathrm{NE}$ & $N E$ & $N E$ \\
\hline Penk1 & No & $\mathrm{NE}$ & $\mathrm{NE}$ & $N E$ & $N E$ \\
\hline Pib5pa & Yes & Yes & Yes & $Y e s(f e w)$ & $N E$ \\
\hline $\operatorname{Scn} 4 \mathrm{~B}$ & Yes & $\mathrm{NE}$ & Yes & $N E$ & $N E$ \\
\hline Scrt1 & Yes & nt (probable) & Yes & Yes (few) & $N E$ \\
\hline Serpini1 & Yes & Yes & Yes & Yes & Yes \\
\hline Slc17a6 & Yes & No & No & $N E$ & $N E$ \\
\hline Slc17a7 & No & Yes & No & $N E$ & $N E$ \\
\hline Spp1 & Yes & No & Yes & $N E$ & $N E$ \\
\hline Tac1 & No & nt (probable) & $\mathrm{NE}$ & Yes (few) & $N E$ \\
\hline Tac2 & No & nt (probable) & $\mathrm{NE}$ & $N E$ & $N E$ \\
\hline Trh & No & $\mathrm{NE}$ & nt (probable) & $N E$ & $N E$ \\
\hline Vamp1 & Yes & Yes & Yes & Yes & Yes (few) \\
\hline
\end{tabular}

Yes/no, experimentally verified by ISH (this study); nt (probable), not tested experimentally, but probably expressed according to the ABA data; NE, not expressed, according to the ABA data and our own experimental observations. For the expression in the hippocampal stratum oriens and the molecular layer of the cerebellum, all data are from the ABA (written in italics).

the dentate gyrus revealed the co-existence of several of the genes found to be expressed in the PV1 nucleus (shown in Fig. 5 for Serpini1, Kcna2 and Pib5pa). As aforementioned for the PV1 nucleus, co-expression was not always apparent within all of the visible Pvalbexpressing cells, and we cannot therefore conclude on the basis of this study that the candidate genes are universally expressed in all Pvalbpositive neurons of the investigated areas. By carefully analyzing the ABA ISH data, we also compared the expression of these genes in the molecular layer of the cerebellum and the hippocampal stratum oriens, both containing mainly Pvalb-positive GABAergic interneurons. Results for the 27 genes are summarized in Table 2. The following conclusions can be made from these observations. The only genes specific for the PV1 nucleus are Slc17a6, Lynx2 and Adcyap1. The genes Crhr1, Scn4b and Spp1 also show a restricted expression pattern, being present in both the glutamatergic PV1 neurons and the GABAergic neurons of the reticular nucleus, but absent from the Pvalb neurons in all of the other areas analyzed. Several genes are expressed in virtually all Pvalb cells analyzed (GABAergic and glutamatergic): Asb13, Gfra1, Hen2, Kcnc1, Kcna2, Serpini1, Vamp1 and Lynx1 (with the exception of the cerebellum for the first four genes).

\section{Serpini1 and Slc17a7 are expressed in the monkey lateral tuberal nucleus}

On the basis of its topography and neuroanatomy, the lateral tuberal nucleus (LTN) in primates was previously suggested to correspond to the PV1 nucleus in rodents (Gerig \& Celio, 2007), although neurochemical homologies have not yet been demonstrated. Indeed, LTN neurons do not express Pvalb but can be revealed with antibodies to somatotostatin (Fig. 6A) (Gerig \& Celio, 2007). We prepared M. fascicularis antisense RNA probes to two genes: one marker of the rodent PV1 nucleus, Serpini1, and one gene encoding vesicular glutamate transporter VGlut1, Slc17a7 (note that only Slc17a6, not Slc17a7, was found to be expressed in the mouse PV1, but, unfortunately, we were unable to obtain probe for Macaca Slc17a6). As presented in Fig. 6, we found a positive expression for both genes in the LTN. Although very preliminary, these observations suggest a possible evolutionary conservation in gene expression between the rodent PV1 and its putative monkey counterpart, the LTN. In particular, the expression of Slc17a7 demonstrates that LTN neurons are also glutamatergic. It will be interesting to analyze the genomewide expression profile of the monkey LTN, in order to determine which of the PV1-expressed genes identified in this study are conserved in their expression pattern in the monkey.

\section{Discussion}

Several conclusions can be drawn from this study: Pvalb-expressing neurons of the PV1 nucleus are glutamatergic, excitatory neurons, which express the Adcyap1 and Nxph4 peptides, but lack classical hypothalamic neuropeptides (including orexin/hypocretin), and share several genes in common with Pvalb-immunoreactive inhibitory, GABA neurons, found in other brain areas.

According to several transcriptomic studies using gene arrays, very few of the genes that were found to be expressed in glutamatergic or 

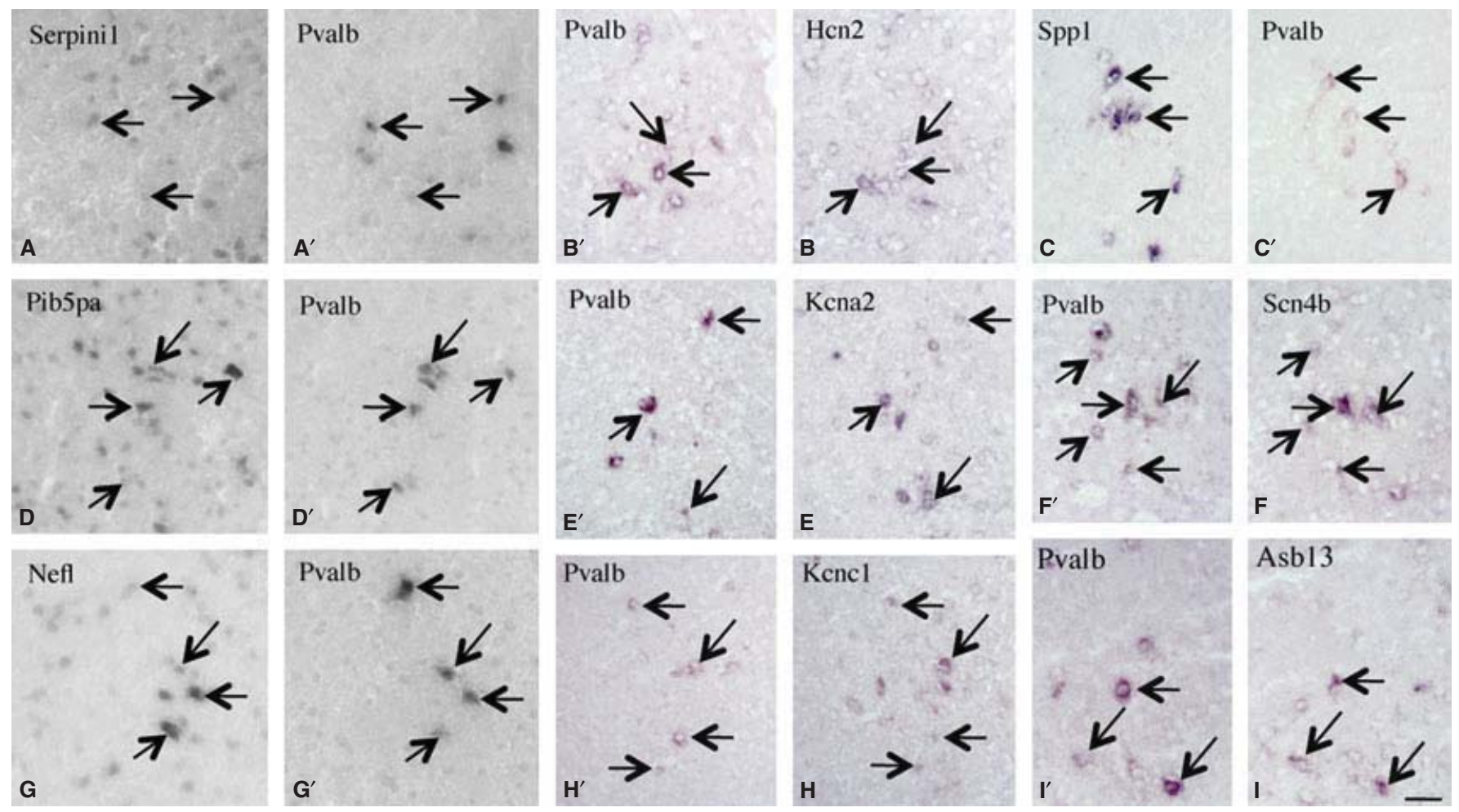

0
0
0
0
0
0
0
0

FIG. 3. Gene expression in the PV1 hypothalamic nuclei. Higher magnification of the PV1 nucleus in adjacent sections stained by ISH for Pvalb (A', B', $C^{\prime}, D^{\prime}, E^{\prime}$, $\mathrm{F}^{\prime}, \mathrm{G}^{\prime}, \mathrm{H}^{\prime}$ and I') and Serpini1 (A), Hcn2 (B), Spp1 (C), Pib5pa (D), Kcna2 (E), Scn4b (F), Nefl (G), Kcnc1 (H) and Asb13 (I). Black arrows point to cells coexpressing Pvalb and the different tested genes. Scale bar: $20 \mu \mathrm{m}$
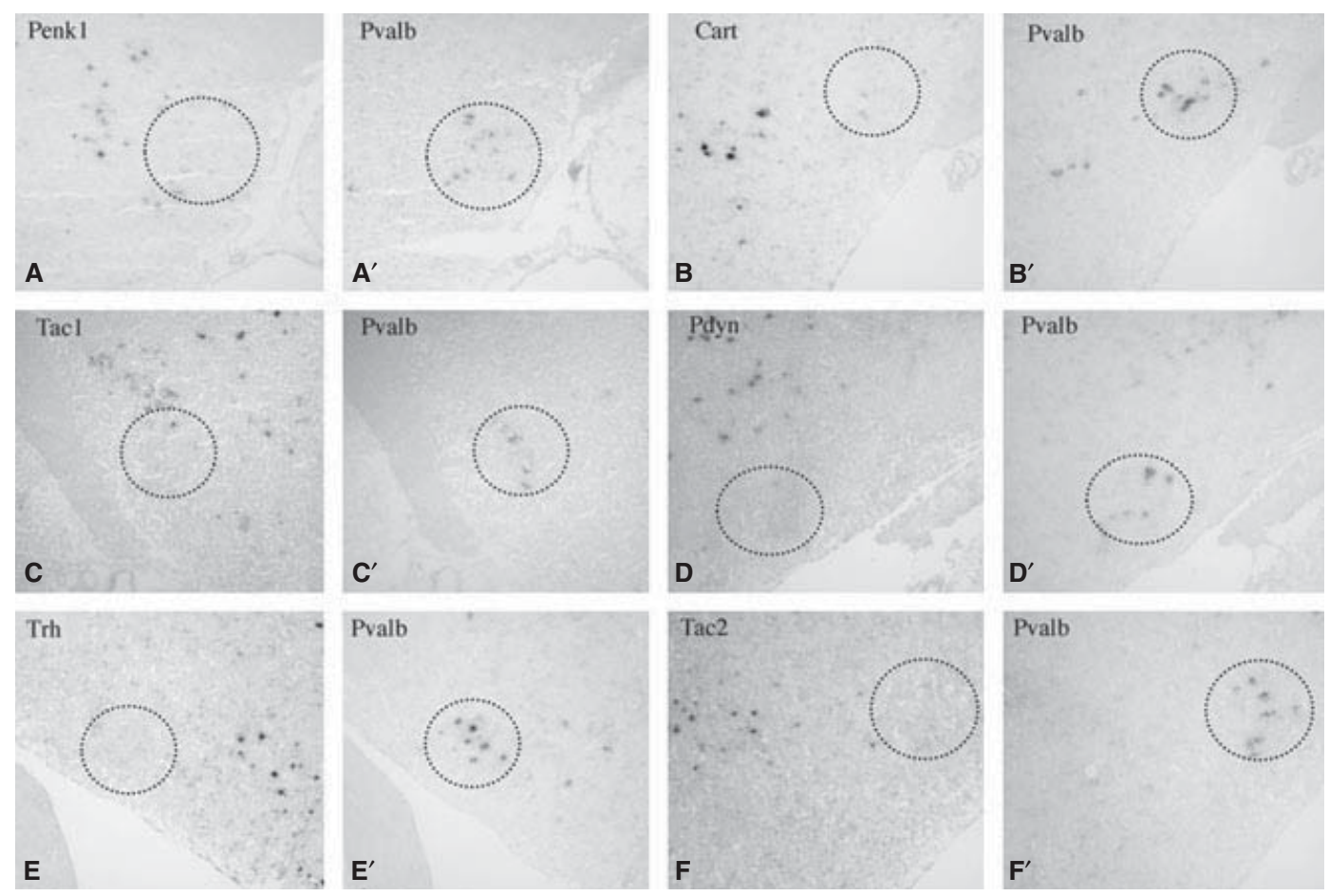

FIG. 4. Various genes encoding neuropeptides/neurohormones located in the far basolateral hypothalamus do not co-express with Pvalb in the PV1 nucleus. Adjacent coronal brain sections stained by ISH for Pvalb $\left(\mathrm{A}^{\prime}-\mathrm{F}^{\prime}\right)$ and various genes encoding hypothalamic neuropeptides/neurohormones: Penk1 (A), Cart (B), Tac1 (C), Pdyn (D), Trh (E) and Tac2 (F). Dashed circles delineate the position of the PV1 nucleus. 

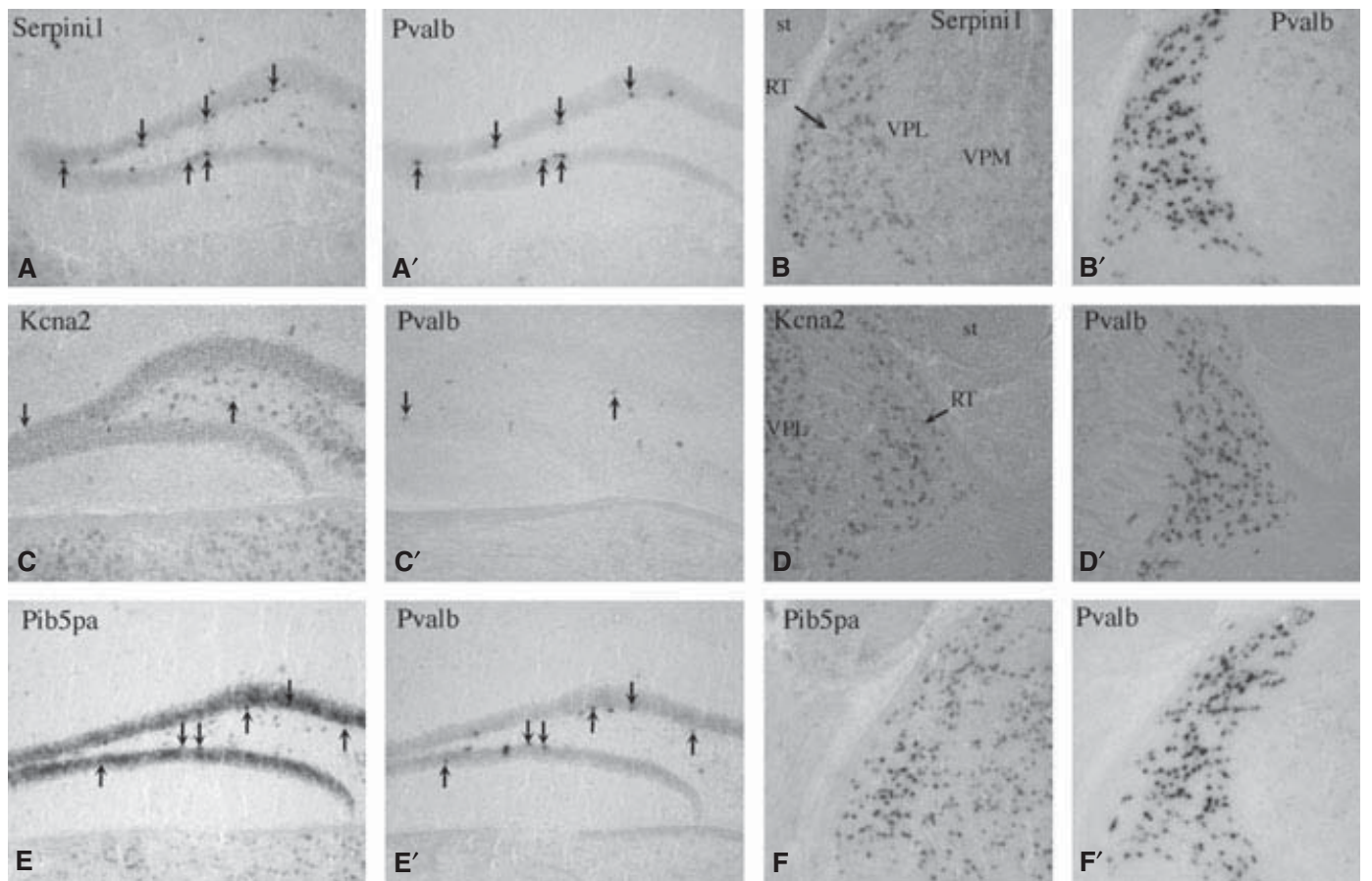

FIG. 5. Most of the genes identified in Pvalb PV1 cells are also expressed with Pvalb in the reticular thalamic nucleus and the dentate gyrus of the hippocampus. Adjacent coronal brain sections stained by ISH for Pvalb $\left(\mathrm{A}^{\prime}-\mathrm{F}^{\prime}\right)$ and Serpini1 (A and B), Kcna2 (C and D) and Pib5pa (E and F). Pictures were taken at the level of the hippocampal dentate gyrus $\left(\mathrm{A}-\mathrm{A}^{\prime}, \mathrm{C}-\mathrm{C}^{\prime}, \mathrm{E}-\mathrm{E}^{\prime}\right)$ and reticular thalamic nucleus $\left(\mathrm{B}-\mathrm{B}^{\prime}, \mathrm{D}-\mathrm{D}^{\prime}, \mathrm{F}-\mathrm{F}^{\prime}\right)$. Black arrows in panels $\mathrm{A}-\mathrm{A}^{\prime}, \mathrm{C}-\mathrm{C}^{\prime}$ and $\mathrm{E}-\mathrm{E}^{\prime}$ highlight cells double stained for Pvalb and the tested genes. RT, reticular nucleus of the thalamus; VPL, ventrolateral nucleus of the thalamus; VPM, ventromedial nucleus of the thalamus; st, stria terminalis.
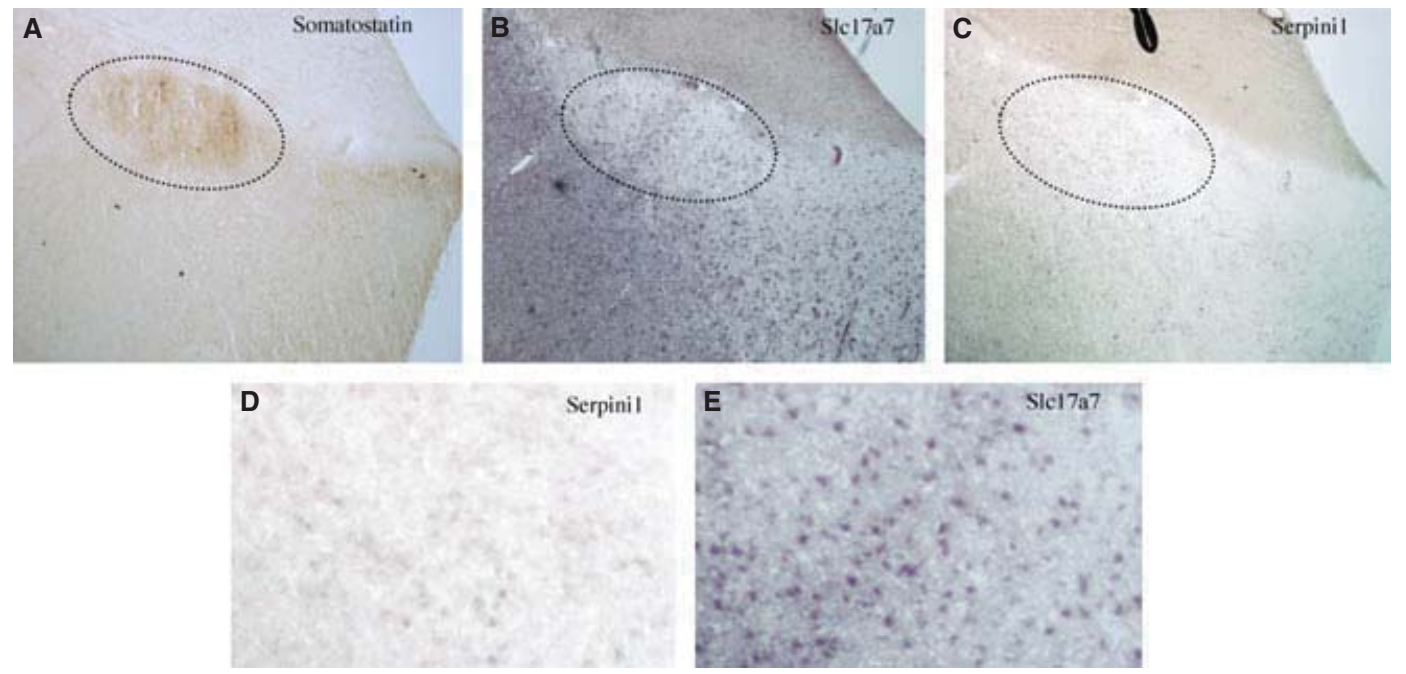

FIG. 6. Conservation of gene expression in the monkey LTN. Macaca fascicularis brain cryosections hybridized with digoxygenin-labeled antisense RNA probes to Slc17a7 (B and E) and Serpini1 (C and D). An adjacent section was immunostained with anti-somatostatin to visualize the LTN (A) (marked with dashed line in $\mathrm{A}-\mathrm{C}$ ). (D and E) Higher magnifications of $\mathrm{B}$ and $\mathrm{C}$, focusing on cells stained within the LTN.

GABAergic neurons (Sugino et al., 2006; Hardt et al., 2008; Subkhankulova et al., 2010) were revealed from the ABA screen. The only exceptions were Kcnc1 (a marker for GABAergic cells) and Nefh and Adcyap1 (reported as typical for glutamatergic cells). Some of the genes expressed in the PV1 nucleus (listed in Table 1) are known to occur in subclasses of GABAergic neurons. These include: Pitx2 (Skidmore et al., 2008; Waite et al., 2011), Scrt (Marin \& Nieto, 2006), Scn1a (Ogiwara et al., 2007; Martin et al., 2010), Gfra1(Pozas \& Ibanez, 2005; Canty et al., 2009), Lynx2 (Dessaud et al., 2006) and
Tmem163 (Burré et al., 2007). Of the tested genes, only Lynx2, Slc17a6 and Adcyap1 were expressed exclusively in the glutamatergic neurons of the PV1 nucleus. These excitatory neurons of the PV1 nucleus thus express many genes in common with inhibitory neurons, but also very specific genes. It would be interesting to isolate Pvalbpositive cells from these different brain areas, and to compare their transcriptome with microarrays.

The PV1 neurons express several ion channels, including the potassium channels Kcna2 (Kv1-2), Kcnc1 (Kv3-1) and Hcn2, and the 
sodium channel Scn4b, and possibly Kcna1 (Kv1-1), Kcnab2, Kcnab3, Kenc2 (Kv3-2), Kcnk1 and Scn1a (Nav1-1). Immunohistological and electrophysiological studies have reported a close relationship between Pvalb and fast-spiking GABAergic neurons in the cerebral cortex (Celio, 1986; Kawaguchi \& Kondo, 2002). Both Kv3.1 and Kv3.2 are found in Pvalb cortical interneurons, and play a crucial role in the generation of the fast-spiking phenotype (Chow et al., 1999). The presence of the same $\mathrm{K}^{+}$channels in neurons of the PV1 nucleus suggests that they also exhibit fast-spiking activity.

Neurons of the PV1 nucleus are devoid of several neuropeptides typical of LHA, including Tac1/2, Penk1, Pdyn, Trh, Cart (this study), and orexin, galanin and melanin-concentrating hormone (Meszar et al., 2011). Adcyap1 is the only exception, together with Nxph4. Adcyap1, also known as pituitary Adcyap, belongs to the vasoactive intestinal peptide (VIP)/glucagon/growth hormone-releasing factor superfamily. Several functions have been attributed to Adcyap1, including the control of food consumption, synaptic plasticity, antinociceptive effects, or hypophysiotrophic activity (Vaudry et al., 2000). Tracing experiments have shown that PV1 neurons are mostly projecting towards the periaqueductal gray and the laterodorsal tegmental nucleus (Celio \& Saper, 1999). As Adcyap1r, the gene encoding an Adcyap1 receptor, is expressed in these two regions (ABA data; see also Vaudry et al., 2000), it could be suggested that Adcyap1, synthesized from PV1 Pvalb-expressing neurons, is released into the laterodorsal tegmental nucleus and periaqueductal gray where it acts by binding to its receptor. Another neuropeptide used by the PV1 neurons might be Nxph4, a member of a family of secreted glycoproteins of which another representative, Nxph1, is expressed primarily in interneurons (Petrenko et al., 1996), and binds to neurexin1 (Missler \& Südhof, 1998). For Nxph4, no receptor has as yet been identified.

Spp1/osteopontin is a secreted glycoprotein, which binds different integrin matrix receptors, and exerts several biological activities including calcium binding, chemotactic effects on astrocytes in the central nervous system, cell adhesion, cell signaling and cell proliferation, and regulation of the ECM (Denhardt et al., 2001). Interestingly, it has an expression pattern in the whole adult mouse brain that closely mimics that of Pvalb (ABA data). Spp1 has to be added to the long list of antigens found in the perineuronal net (Celio \& Blumcke, 1994; Celio et al., 1998), a special ECM rich in proteoglycans and glycoproteins (Bonneh-Karkay \& Wiley, 2009). The Serpinil brain expression pattern is also very similar to that of Pvalb. Serpini1/Neuroserpin is an axonally secreted peptidase inhibitor of the serpin family. It is believed to be a critical regulator of extracellular proteolytic events associated with synaptic plasticity and regeneration, through the inhibition of tissue plasminogen activator, a major serine protease of the brain ECM (Krueger et al., 1997; Bonneh-Karkay \& Wiley, 2009). The ECM is particularly accentuated around Pvalb neurons (Meszar et al., 2011). Thus, Spp1 and Serpinil might be involved in shaping a peculiar ECM around neurons expressing Pvalb.

Lynx 1 and Lynx2 belong to a family of prototoxins that bind to and regulate the functions of nicotinic acetylcholine receptors, both in vivo and in vitro (Ibanez-Tallon et al., 2002; Miwa et al., 2006; Tekiney et al., 2009). Lynx1/2 are glycosylphosphatidylinositol (GPI)anchored proteins, which physically associate with their receptors in the somatodendritic membranes of many neurons (Dessaud et al., 2006). Neurons of the PV1 nucleus express both Lynx1 and Lynx2 (our ISH data), and possibly Chrnb2 and Chrm2 (ABA data), and receive Chat-positive terminals (our unpublished data), suggesting that modulation of cholinergic receptors might be important for their functioning.
In conclusion, our study illustrates the power of genome-wide ISH results to generate hypotheses, and the importance of higher resolution sequential ISH to determine gene batteries expressed in small numbers of cells in the brain. We expect this approach to be generally applicable to refining neuroanatomy and gaining functional insight into other small brain regions.

\section{Supporting Information}

Additional supporting information can be found in the online version of this article:

Table S1. Genes found by both ALLENMINER searches: (1) PV1 enrichment and (2) Pvalb-like PV1 expression.

Table S2. ALLENMINER results for genes with Pvalb-like expression in PV1.

Table S3. ALLENMINER results for enriched in PV1.

Table S4. Genes found by either ALLENMINER search: (1) PV1 enrichment or (2) Pvalb-like expression similarity.

Please note: As a service to our authors and readers, this journal provides supporting information supplied by the authors. Such materials are peer-reviewed and may be re-organized for online delivery, but are not copy-edited or typeset by Wiley-Blackwell. Technical support issues arising from supporting information (other than missing files) should be addressed to the authors.

\section{Acknowledgements}

We thank Dr Bruno Muller for advice on the design of ISH experiments, Simone Eichenberger for animal care, and Véronique Piccand for technical help. We also thank Dr Friedrich Raulf for the gift of monkey brain RNA and tissue. This work was supported by a Swiss National Foundation grant (NF 3100A0-113524) and a Novartis Foundation grant.

\section{Abbreviations}

ABA, Allen Brain Atlas; ECM, extracellular matrix; ISH, in situ hybridization; LHA, lateral hypothalamic area; LTN, lateral tuberal nucleus; Pvalb, parvalbumin; SSC, saline sodium citrate buffer; VGlut2, vesicular glutamate transporter 2 .

\section{References}

Baimbridge, K., Celio, M. \& Rogers, J. (1992) Calcium binding proteins in the nervous system. Trends Neurosci., 15, 303-308.

Berthoud, H.R. \& Münzberg, H. (2011) The lateral hypothalamus as integrator of metabolic and environmental needs: from electrical self-stimulation to opto-genetics. Physiol. Behav., 104, 29-39.

Bonneh-Karkay, D. \& Wiley, C. (2009) Brain extracellular matrix in neurodegeneration. Brain Pathol., 19, 573-585.

Burré, J., Zimermann, H. \& Volknandt, W. (2007) Identification and characterization of SV31, a novel synaptic vesicle membrane protein and potential transporter. J. Neurochem., 103, 276-287.

Canty, A.J., Dietze, J., Harvey, M., Enomoto, H., Milbrandt, J. \& Ibanez, C.F. (2009) Regionalized loss of parvalbumin interneurons in the cerebral cortex of mice with deficits in GFRalphal signaling. J. Neurosci., 29, 1069510705.

Celio, M. (1986) Parvalbumin in most gamma-aminobutyric acid-containing neurons of the rat cerebral cortex. Science, 231, 995-997.

Celio, M. (1990) Calbindin $28 \mathrm{~K}$ and parvalbumin in the rat nervous system. Neuroscience, 35, 375-475.

Celio, M. \& Blumcke, I. (1994) Perineuronal nets - a specialized form of extracellular matrix in the adult nervous system. Brain Res. Brain Res. Rev., 19, 128-145.

Celio, M. \& Saper, C. (1999) The Parvalbumin Positive Nucleus of the Lateral Hypothalamus. Neuroscience 1999 Abstract. Society for Neuroscience, San Diego. 
Celio, M., Spreafico, R., DeBiasi, S. \& Vitellaro-Zucarello, L. (1998) Perineuronal nets: past and presents. Trends Neurosci., 21, 510-515.

Chow, A., Erisir, A., Farb, C., Nadal, M., Ozaita, A., Lau, D., Welker, E. \& Rudy, B. (1999) $\mathrm{K}^{+}$channel expression distinguishes subpopulations of parvalbumin and somatostatin containing neocortical interneurons. J. Neurosci., 19, 9332-9345.

Davis, F. \& Eddy, S. (2009) A tool for identification of genes expressed in patterns of interest using the Allen Brain Atlas. Bioinformatics, 25, 1647-1654.

Denhardt, D., Noda, M., O’Regan, A., Pavlin, P. \& Berman, J. (2001) Osteopontin as a means to cope with environmental insults: regulation of inflammation, tissue remodeling and cell survival. J. Clin. Invest., 107, 1055-1061.

Dessaud, E., Salaun, D., Gayet, O., Chabbert, M. \& De Lapeyriere, O. (2006) Identification of Lynx2, a novel member of the ly-6/neurotoxin superfamily, expressed in neuronal subpopulations during mouse development. Mol. Cell. Neurosci., 31, 232-242.

Dong, H., Swanson, L., Chen, L., Fanselow, M. \& Toga, A. (2009) Genomicanatomic evidence for distinct functional domains in hippocampal field CA1. Proc. Natl. Acad. Sci. USA, 106, 11794-11799.

D’Souza, C., Chopra, V., Varhol, R., Xie, Y., Bohacec, S., Zhao, Y., Lee, L., Bilenky, M., Portales-Casamar, E., He, A., Wasserman, W., Goldowitz, D. Marra, M., Holt, R., Simpson, E. \& Jones, S. (2008) Identification of a set of genes showing regionally enriched expression in the mouse brain. BMC Neurosci., 9, 66.

Gerig, A. \& Celio, M. (2007) The human lateral tuberal nucleus: immunohistochemical characterization and analogy to the rodent PV1 nucleus. Brain Res., 1139, 110-116.

Hardt, O., Scholz, C., Kusters, D., Yanagawa, Y., Pennartz, S., Cremer, H. \& Bosio, A. (2008) Gene expression analysis defines differences between region specific GABAergic neurons. Mol. Cell. Neurosci., 39, 418-428.

Ibanez-Tallon, I., Miwa, J., Wang, H., Adams, N., Crabtree, G., Sine, S. \& Heintz, N. (2002) Novel modulation of neuronal nicotinic acetylcholine receptors by association with the endogenous prototoxin Lynx1. Neuron, 33, 893-903.

Kawaguchi, Y. \& Kondo, S. (2002) Parvalbumin, somatostatin and cholecystokinin as chemical markers for specific GABAergic interneuron types in the rat frontal cortex. J. Neurocytol., 31, 277-287.

Krueger, S., Ghisu, G., Cinelli, P., Gschwend, T., Osterwalder, T., Wolfer, D. \& Sonderegger, P. (1997) Expression of neuroserpin, and inhibitor of tissue plasminogen activator in the developing and adult nervous system of the mouse. J. Neurosci., 17, 8984-8996.

Lein, E., Hawrylycz, M., Ao, N., Ayres, M., Bensinger, A., Bernard, A., Boe, A., Boguski, M., Brockway, K., Byrnes, E., Chen, L., Chen, L., Chen, T., Chin, M., Chong, J., Crook, B., Czaplinska, A., Dang, C., Datta, S., Dee, N., Desaki, A., Desta, T., Diep, E., Dolbeare, T., Donelan, M., Dong, H., Dougherty, J., Duncan, B., Ebbert, A., Eichele, G., Estin, L., Faber, C., Facer, B., Fields, R., Fischer, S., Fliss, T., Frensley, C., Gates, S., Glattfelder, K., Halverson, K., Hart, M., Hohmann, J., Howell, M., Jeung, D., Johnson, R., Karr, P., Kawal, R., Kidney, J., Knapik, R. \& Kuan, C., Lake, J., Laramee, A., Larsen, K., Lau, C., Lemon, T., Liang, A., Liu, Y., Luong, L. Michaels, J., Morgan, J., Morgan, R., Mortrud, M., Mosqueda, N., Ng, L., Ng, R., Orta, G., Overly, C., Pak, T., Parry, S., Pathak, S., Pearson, O., Puchalski, R., Riley, Z., Rockett, H., Rowland, S., Royall, J., Ruiz, M., Sarno, N., Schaffnit, K., Shapovalova, N., Sivisay, T., Slaughterbeck, C., Smith, S., Smith, K., Smith, B., Sodt, A., Stewart, N., Stumpf, K., Sunkin, S., Sutram, M., Tam, A., Teemer, C., Thaller, C., Thompson, C., Varnam, L., Visel, A., Whitlock, R., Wohnoutka, P., Wolkey, C., Wong, V., Wood, M., Yaylaoglu, M., Young, R., Youngstrom, B., Yuan, X., Zhang, B., Zwingman, T. \& Jones, A. (2007) Genome wide atlas of gene expression in the adult mouse brain. Nature, 445, 168-176.

Leinninger, G.M. (2011) Lateral thinking about leptin: a review of leptin action via the hypothalamus. Physiol. Behav., 104, 572-581.
Marin, F. \& Nieto, M. (2006) The expression of Scratch genes in the developing and adult brain. Dev. Dyn., 235, 2586-2591.

Martin, M., Dutt, K., Papale, L., Dube, C., Dutton, S., De Haan, G., Shankar, A., Tufik, S., Meisler, M., Baram, T., Goldin, A. \& Escayg, A. (2010) Altered function of the SCN1A voltage-gated sodium channel leads to gamma-aminobutyric acid-ergic (GABAergic) interneuron abnormalities. J. Biol. Chem., 285, 9823-9834.

Meszar, Z., Girard, F., Saper, C. \& Celio, M. (2011) The lateral hypothalamic parvalbumin immunoreactive (PV1) nucleus in rodents. J. Comp. Neurol., doi: 10.1002/cne.22789 [Epub ahead of print].

Missler, M. \& Südhof, T. (1998) Neurexophilins form a conserved family of neuropeptide-like glycoproteins. J. Neurosci., 18, 3630-3638.

Miwa, J., Stevens, T., King, S., Caldorone, B., Ibanez-Tallon, I., Xiao, C., Fitzsimmonds, R., Pavlides, C., Lester, H., Picciotto, M. \& Heintz, N. (2006) The prototoxin Lynx1 acts on nicotinic acetylcholine receptors to balance neuronal activity and survival in vivo. Neuron, 51, $587-600$.

Ogiwara, I., Miyamoto, H., Morita, N., Atapour, N., Mazaki, E., Inoue, I., Takeuchi, T., Itohara, S., Yanagawa, Y., Obata, K., Furuichi, T., Hensch, T. \& Yamakawa, K. (2007) Na(v)1.1 localizes to axons of parvalbumin positive inhibitory interneurons: a circuit basis for epileptic seizures in mice carrying an Scn1a mutation. J. Neurosci., 27, 5903-5914.

Olszewski, P., Cedernaes, J., Olsson, F., Levine, A. \& Schioth, H. (2008) Analysis of the network of feeding neuroregulators using Allen Brain Atlas. Neurosci. Biobehav. Rev., 32, 945-956.

Petrenko, A., Ullrich, B., Missler, M., Krasnoperov, V., Rosahl, T. \& Sudhof, T. (1996) Structure and evolution of neurexophilin. J. Neurosci., 16, 4360 4369

Pozas, E. \& Ibanez, C. (2005) GDNF and GFRalpha1 promote differentiation and tangential migration of GABAergic neurons. Neuron, 45, 701-713.

Sakurai, T., Mieda, M. \& Tsujino, N. (2010) The orexin system: roles in sleep/wake regulation. Ann. NY Acad. Sci., 1200, 149-161.

Schwaller, B. (2009) The continuing disappearance of pure $\mathrm{Ca}^{2+}$ buffers. Cell. Mol. Life Sci., 66, 275-300.

Skidmore, J., Cramer, J., Martin, J. \& Martin, D. (2008) Cre fate mapping reveals lineage specific defects in neuronal migration with loss of Pitx2 function in the developing hypothalamus and subthalamic nucleus. Mol. Cell. Neurosci., 37, 696-707.

Subkhankulova, T., Yano, K., Robinson, H. \& Livesey, F. (2010) Grouping and classifying electrophysiologically defined classes of neocortical neurons by single cell, whole genome expression profiling. Front. Mol. Neurosci., 3, 10 .

Sugino, K., Hempel, C., Miller, M., Hattox, A., Shapiro, P., Wu, C., Huang, Z. \& Nelson, S. (2006) Molecular taxonomy of major neuronal classes in the adult mouse forebrain. Nat. Neurosci., 9, 99-107.

Takamori, S., Rhee, J., Rosenmund, C. \& Jahn, R. (2000) Identification of a vesicular glutamate transporter that defines a glutamatergic phenotype in neurons. Nature, 407, 189-194.

Tekiney, A., Nong, Y., Miwa, J., Lieberam, I., Ibanez-Tallon, I., Greengard, P. \& Heintz, N. (2009) A role for Lynx2 in anxiety-related behavior. Proc. Natl. Acad. Sci. USA, 106, 4477-4482.

Thompson, C., Pathak, S., Jeromin, A., Ng, L., MacPherson, C., Mortrud, M., Cusick, A., Riley, Z., Sunkin, S., Bernard, A., Puchalski, R., Gage, F., Jones, A., Bajic, V., Hawrylycz, M. \& Lein, E. (2008) Genomic anatomy of the hippocampus. Neuron, 60, 1010-1021.

Vaudry, D., Gonzalez, B., Basille, M., Yon, L., Fournier, A. \& Vaudry, H. (2000) Pituitary adenylate cyclase-activating polypeptide and its receptors: from structure to functions. Pharmacol. Rev., 52, 269-324.

Waite, M., Skidmore, J., Billi, A., Martin, J. \& Martin, D. (2011) GABAregic and glutamatergic identities of developing midbrain Pitx2 neurons. Dev. Dyn., 240, 333-346. 